EPJ Web of Conferences 61, 03007 (2013)

DOI: $10.1051 /$ epjconf/ 20136103007

(C) Owned by the authors, published by EDP Sciences, 2013

\title{
Evidence for quiescent synchrotron emission in the black hole X-ray transient Swift J1357.2-0933
}

\author{
T. Shahbaz ${ }^{1,2, a}$, D.M. Russell ${ }^{1,2}$, C. Zurita ${ }^{1,2}$, J. Casares $^{1,2}$, J.M. Corral-Santana ${ }^{1,2}$, V.S. Dhillon ${ }^{3}$, and T.R. Marsh ${ }^{4}$ \\ ${ }^{1}$ Instituto de Astrofísica de Canarias (IAC), E-38200 La Laguna, Tenerife, Spain \\ ${ }^{2}$ Dept. Astrofísica Universidad de La Laguna (ULL), E-38206 La Laguna, Tenerife, Spain \\ ${ }^{3}$ Department of Physics and Astronomy, University of Sheffield, Sheffield, S3 7RH, UK \\ ${ }^{4}$ Department of Physics, University of Warwick, Coventry CV4 7AL, UK
}

\begin{abstract}
.
We present high time-resolution optical and infrared observations of the edge-on black hole X-ray transient Swift J1357.2-0933. Our data taken in 2012 shows the system to be at its pre-outburst magnitude and so the system is in quiescence. In contrast to other X-ray transients, the quiescent light curves of Swift J1357.2-0933 do not show the secondary star's ellipsoidal modulation. The optical and infrared light curves is dominated by variability with an optical fractional rms of about 20 per cent, much larger than what is observed in other systems. The quiescent ultraviolet to mid-IR spectral energy distribution in quiescence is dominated by a nonthermal component with a power-law index of -1.4, (the broad-band rms SED has a similar index) which arises from optically thin synchrotron emission from a jet; the lack of a peak in the spectral energy distribution rules out advection-dominated models (based on [19]).
\end{abstract}

\section{Observations}

Multi-colour photometric observations of Swift J1357.20933 were obtained with ULTRACAM [3] on the 4.2$\mathrm{m}$ William Herschel Telescope atop La Palma during the nights of UT 2012 April 24 and 25. On UT 2012 April 24 observations were taken using the Sloan $u^{\prime}-, g^{\prime}$ - and $r^{\prime}$-band filters, whereas on the 2012 April 25 observations were taken using the Sloan $u^{\prime}-, g^{\prime}$ - and $i^{\prime}$-band filters. An exposure time of $10 \mathrm{~s}$ was used for the $g^{\prime}-, r^{\prime}$ - and $i^{\prime}$-bands, however, given the faintness of the target an exposure time of $20 \mathrm{~s}$ was used in the $u^{\prime}$-band. Infrared (IR) $J, H$ and $K_{S}$ images of Swift J1357.2-0933 were taken with the 2.5$\mathrm{m}$ Nordic Optical telescope with the near-IR Camera and spectrograph (NOTCam) on three different epochs. On UT 2012 January 9, UT 2013 February 3 and 2013 March 23. The resulting optical and IR magnitudes of Swift J1357.20933 are given in Table 1.

\section{The optical/infrared light curve}

In comparison with other light curves of quiescent $\mathrm{X}$ ray transients we would expect the optical light curve of Swift J1357.2-0933 to show the secondary star's ellipsoidal modulation with superimposed flare events. However, as one can see in Fig. 1 our Sloan-band optical light curves taken on UT 2012 April 24 and 25 only show large amplitude flares, up to $\sim 1.5$ mag superimposed on a flat

\footnotetext{
a e-mail: tsh@iac.es
}

Table 1. Mean observed quiescent magnitudes.

\begin{tabular}{lcc}
\hline Date UT & Band & mean mag. \\
& & \\
\hline 2012 January 9 & J & $19.38 \pm 0.28$ \\
2012 April 24 & u' & $23.12 \pm 0.04$ \\
2012 April 24 & g' & $22.26 \pm 0.38$ \\
2012 April 24 & r' & $21.54 \pm 0.35$ \\
2012 April 25 & i' & $21.21 \pm 0.36$ \\
2013 February 3 & J & $19.60 \pm 0.06$ \\
2013 February 3 & H & $19.73 \pm 0.05$ \\
2013 February 3 & $\mathrm{K}_{s}$ & $18.24 \pm 0.04$ \\
2013 March 24 & J & $19.38 \pm 0.06$ \\
2013 March 24 & H & $19.02 \pm 0.05$ \\
2013 March 25 & J & $19.17 \pm 0.05$ \\
2013 March 25 & H & $18.68 \pm 0.05$ \\
2013 March 25 & $\mathrm{K}_{s}$ & $18.14 \pm 0.05$ \\
\hline
\end{tabular}

light curve. There is no evidence for any orbital modulation which suggests that another source of light with large variability completely dominates the secondary star's optical flux. The fractional rms variability of the optical flaring activity is large, $\sim 35$ per cent in $g^{\prime}-, r^{\prime}$ - and $i^{\prime}$-bands, more than a factor of 3 larger than what is normally observed in the optical light curves of quiescent X-ray transients with similar time resolution ([17], [18]), and similar to the X-ray fractional rms seen in the hard state of X-ray binaries. Similarly the NOTCam $J$-band light curve taken on UT 2012 January 9 which has a duration of $2.6 \mathrm{~h}$ also 
SWIFT J1357.2-0933 (24 April 2012)
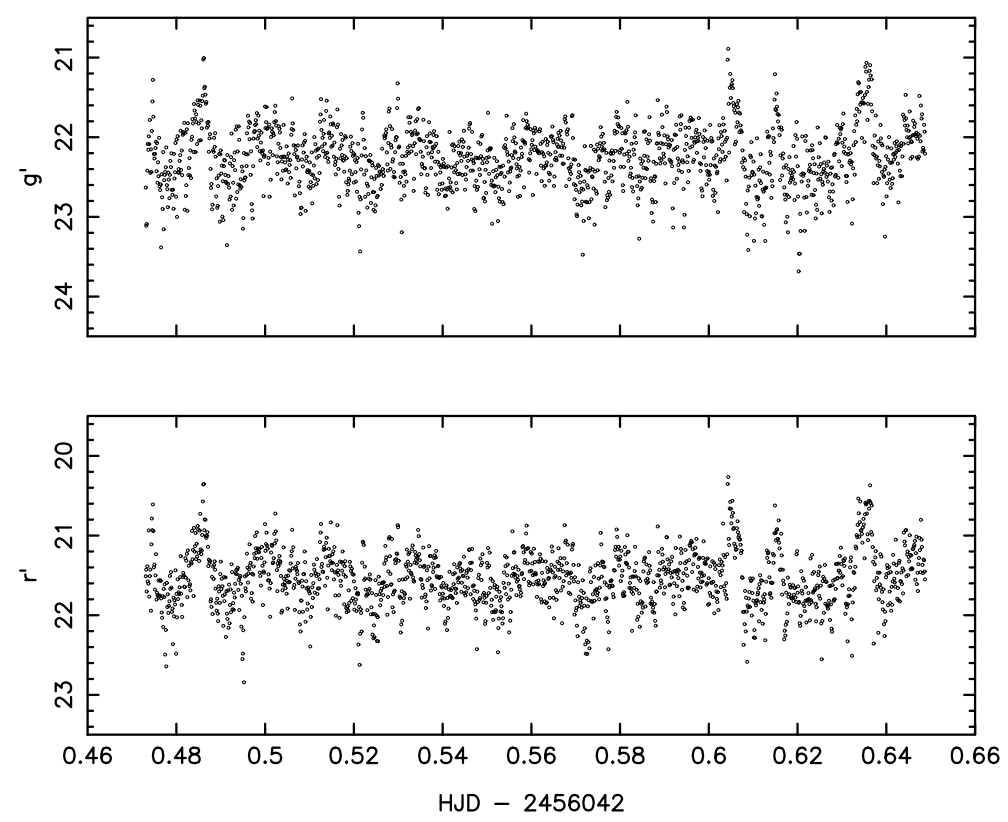

Figure 1. The observed ULTRACAM Sloan-band light curves of J1357.2-0933 taken on UT 2012 April 24.

shows no evidence of the secondary star's ellipsoidal modulation. The light curve is dominated by variability with a fractional rms of $\sim 23$ per cent and flare events lasting 1030 min with amplitudes of $\sim 2$ mag are observed.

In Fig. 1 flare events lasting 2-10 min are seen as well as numerous rapid flare events which are not resolved. There are also a number of $\sim 0.8 \mathrm{mag}$ "dip" events observed on both nights which look similar to the dips seen in outburst, but less frequent [1]. The dips have a characteristic duration of $\sim 2$ min and drop in brightness by up to $\sim 0.5-$ $1.0 \mathrm{mag}$, which is equivalent to a factor $\sim 2$ reduction in flux, similar to what is observed during outburst. The recurrence period of the dips is $\sim 30 \mathrm{~min}$, a factor of $\sim 10$ longer than what is observed in outburst [1].

\section{The observed spectral energy distribution}

Pre-outburst SDSS DR7 images taken on 2006 May 26 show Swift J1357.2-0933 to be at $g^{\prime}=22.8$ [12]. Our postoutburst ULTRACAM data taken on 2012 April 24 and 25 show the system to be at $g^{\prime}=22.3$ and so given the large up to $\sim 1.5 \mathrm{mag}$ intrinsic variability observed, we can assume that the ULTRACAM 2012 and SDSS DR7 2006 datasets were taken when the target was in the same state. Similarly the NOTCam data taken in 2012 and 2013 have similar flux levels and so the target is in the same state. In the WISE catalogue [22], there are detections of Swift J1357.2-0933 taken on 2010 January 21 at $3.4 \mu \mathrm{m}$ (W1), $4.6 \mu \mathrm{m}$ (W2), $12 \mu \mathrm{m}$ (W3) and $22 \mu \mathrm{m}$ (W4; upper limit), a year before the outburst. Plotting the WISE, NOTCam and ULTRACAM fluxes we can see that the WISE data are a power-law extension of the quiescent optical/IR fluxes (see Fig. 2). Given the fact that the 2012 optical/IR data and the 2010 WISE data can be represented by the same power-law model, it is safe to assume that the system was in the same state when these observations were taken i.e. in quiescence. Fitting the ULTRACAM(4 points), NOTCam (6 points) and WISE data (3 points) with a power-law model gives an index $\alpha=-1.4 \pm 0.1$. We also tried fitting the data with a two-component model; powerlaw plus M4 star taken from IRAF/SYNPHOT; the radius of the M4 star was fixed at $0.29 \mathrm{R}_{\odot}[1]$ and the distance was left as a free parameter. However, an F-test shows that the two-component model is only better than the single component model at 0.05 per cent confidence level. Hence the broad-band mid-IR to UV flux SED is best de scribed by a single power-law model.

\section{Discussion}

We can compare the flares in Swift J1357.2-0933 to other quiescent black hole XRTs. Swift J1357.2-0933 displays the largest amplitude variability seen in any quiescent XRT reported to date; it shows large flaring up to $\sim 1-2$ mag in amplitude. Given the power-law index for the SED and the high inclination angle (which minimises any dilution from the disc) this tells us that any thermal component i.e. from the secondary star or accretion disc/rim is heavily diluted and it is safe to assume that the observed variability originates in the non-thermal emission. In the following we compare the observed quiescent SED with what is expected from an accretion disc/flow and a jet.

\subsubsection{A jet?}

Here we consider the possibility that the quiescent nearIR-optical SED that can be fitted by a single power-law 


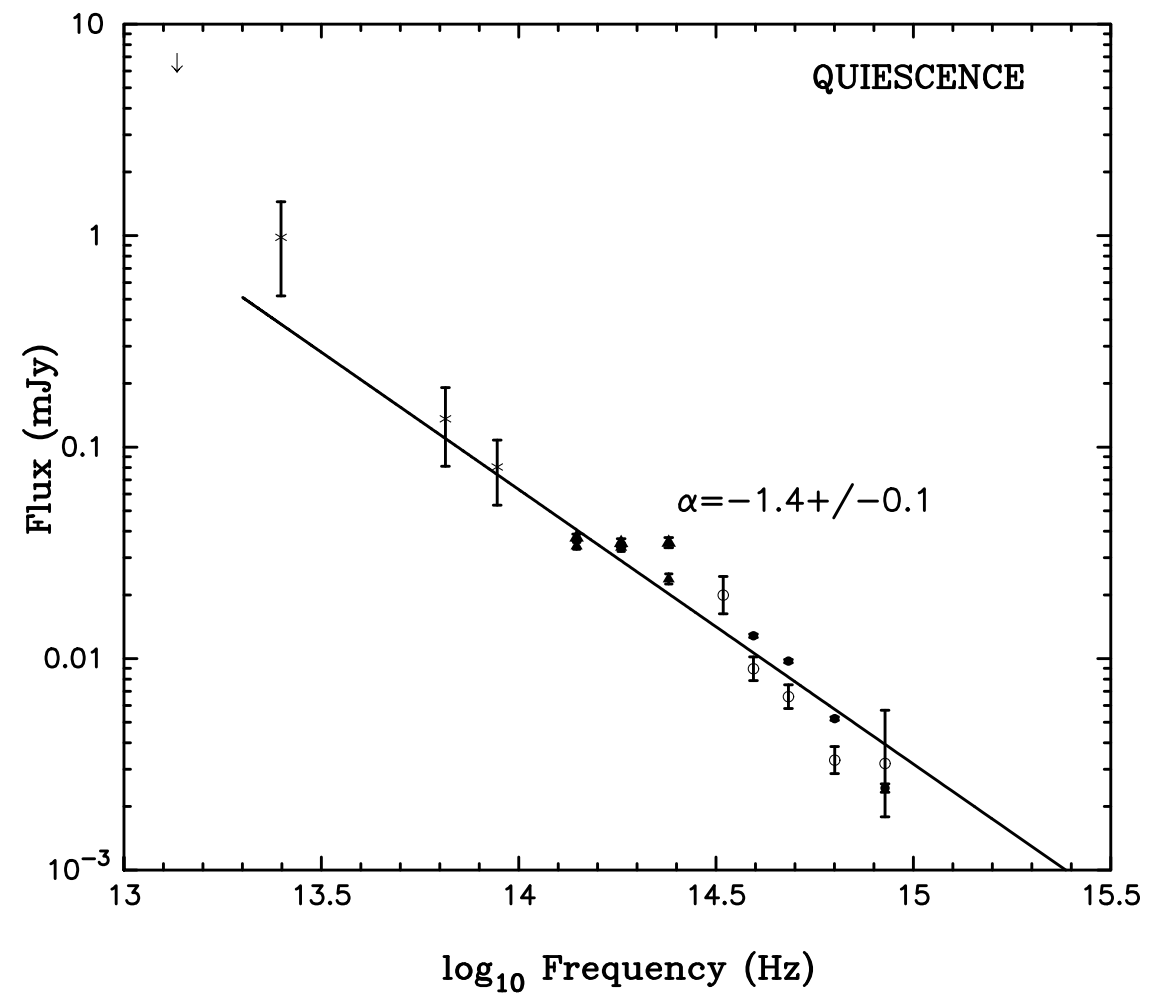

Figure 2. The dereddened spectral energy distribution taken during quiescence. The ULTRACM optical data taken on 2012 April $24-$ 25 and the NOTCam $J, H$ and $K s$-band data taken on 2012 February 2 are shown as filled circles and triangles respectively. The open circles show the SDSS DR7 images taken on 2006 May 26 [12], and confirms that the source was in the same state when the independent datasets were taken. The stars show the WISE data (W1-W4) taken on 2010 January 21 [22]. The solid line is a power-law fit to the ULTRACAM, NOTCam and WISE data and implies that the same mechanism produces the optical and near-IR flux.

of index $\alpha=-1.4$ (which is similar to the IR-optical rms SED) could also be interpreted as emission from a jet in the system. For optically thin synchrotron emission the only parameter which varies the spectral index is the particle energy distribution of the emitting electrons, $\alpha_{\text {thin }}=$ $(1-p) / 2$. If the observed quiescent power law index of $\alpha=-1.4$ is interpreted as optically thin synchrotron, the particle energy distribution is much higher than usually observed in black hole XRBs and AGN, $p=3.8$ (typical values are $2 \leq p \leq 3$ ). However, a steeper spectral index could be explained by a thermal, possibly Maxwellian distribution of electrons in a weaker jet. In one case, the spectral index of the jet emission in XTE J1550-564 was seen to vary smoothly from $\alpha=-1.5$ when the jet was fainter, to $\alpha=-0.6$ when the jet was brighter, and was interpreted in this way [13]. Some optically thin SEDs of XTE J1118+480 also have similar steeper spectral indices [14]. Some low luminosity AGN also have steep synchrotron spectra, with $-3<\alpha<-1$ [7]. In these sources the optically thick-thin spectral break resides in the (sub)mm to mid-IR regime, and the optically thin synchrotron emission above the break curves down to this steep SED. Magnetohydrodynamical numerical simulations are able to reproduce the steep spectra as radiatively cooled synchrotron emission, where the spectral shape is dependent on the configuration of the magnetic field in the inner regions [4]. It may be the case that the faint, quiescent spectrum of Swift J1357.2-0933 could be explained by a popu- lation of sub-relativistic electrons in a weak outflow. This would imply that the break in the jet spectrum between the 'flat' or inverted, partially self-absorbed radio power-law and the optically thin power-law must reside at lower frequencies than the W3 WISE bandpass, $v_{\text {break }}<2.5 \times 10^{13}$ Hz. Theoretically, this break is expected to shift to lower frequencies at lower luminosities, if other parameters are unchanged [6], and this constraint is consistent with this picture since breaks have been detected at higher frequencies in outburst [8]. Observationally, other parameters appear to change, which is likely to produce the wide scatter observed in the relation between jet break frequency and luminosity in black hole XRBs [14].

The reason why this outflow dominates the emission in this source and not other quiescent black hole XRBs is likely to be related to the inclination to our line of sight. The projected disc surface area is much smaller for this edge-on black hole XRB, but if the inner jet is not eclipsed by the disc or the star, and if beaming effects are negligible, the ratio of jet to disc emission will be greater. To summarize, the observed steep power-law, with high amplitude flickering is consistent with a variable, weak jet, and other interpretations are most likely ruled out (see below). 


\subsubsection{An accretion disc?}

[10] have shown that the X-ray observations of quiescent XRTs can be explained by a two-component accretion flow model. The geometry of the flow consists of a hot inner advection-dominated accretion flow (ADAF) that extends from the black hole horizon to a transition radius and a thin accretion disc that extends from the transition radius to the outer edge of the accretion disc. In principle, interactions between the hot inner ADAF and the cool, outer thin disc, at or near the transition radius, can be a source of optical variability [5]. It has been suggested that the flares observed in quiescent X-ray transients arise from the transition radius [15, 21]. Indeed, QPO and low-frequency break features in the power spectra have been used to determine the transition radius, the transition between the thin and advective disc (ADAF) regions [9, 18].

In the original ADAF models the optical flux is produced by synchrotron emission by the hot electrons in the ADAF [9]. However, as pointed out in [15], it is difficult to explain the flare spectrum in terms of optically thin synchrotron emission, unless the electrons follow a much steeper power-law electron energy distribution compared to solar flares. The SED index of the flares in the optical has now been determined in several systems, in Swift J1357.2-0933 $\alpha \sim-1.4$ (see section 3), A0620-00 ( $\alpha \sim-1.6$; [16]), GU Mus $(\alpha \sim-1.2$; [18]) and XTE J1118+480 (there is no index value but the $i^{\prime}$-band flux per unit frequency interval is larger than that in the $g^{\prime}$-band; [17]). These indices are much steeper than the electron energy distribution in solar and stellar flares; for solar and stellar flares the power-law index of the electron energy distribution $\sim 2$ [2], which corresponds to a SED with an index $\alpha=-0.5$. However, it should be noted that in the ADAF models, there are three radiation processes that are important: synchrotron emission, Compton scattering, and bremsstrahlung, each of which produce distinct and easily recognized features in the quiescent SED. The thermal synchrotron emission in ADAFs is invariably self-absorbed and produces a sharp cutoff peak, with a peak frequency that depends on the mass of the black hole: $v_{s} \sim 10^{15}\left(M / M_{\odot}\right)^{-1 / 2} \mathrm{~Hz}$. The synchrotron peak is in the optical band for stellar-mass black holes. Synchrotron emission from different radii in the flow occurs at different frequencies and the peak emission, however, always originates close to the black hole and reflects the properties of the accreting gas near the Schwarzschild radius of the black hole [20]. For Swift J1357.2-0933 with a $\sim 10 \mathrm{M}_{\odot}$ black hole the peak frequency would lie at $10^{14.5} \mathrm{~Hz}$. Such a peak is not seen in quiescent SED of Swift J1357.20933.

\section{References}

[1] Corral-Santana J., et al., Science, 339, 6123, 1048, (2013)

[2] Crosby N.B., Aschwanden M.J., Schmitt J.H.M.M., Sol.Phys, 143, 275, (1993)

[3] Dhillon V., et al., MNRAS, 378, 825, (2007)

[4] Drappeau S., Dibi S., Dexter J., Markoff S., Fragile P. C., MNRAS, 431, 2872, (2013)

[5] Esin A. A., McClintock J.E., Narayan R., ApJ, 489, 865, (1997)

[6] Falcke, H., Körding, E., \& Markoff, S., A\&A, 414, 895, (2004)

[7] Fernández-Ontiveros J. A., Prieto M. A., AcostaPulido J. A., Montes M., JPhCS, 372, 012006, (2012)

[8] Gandhi, P., Blain, A. W., Russell, D. M., et al., ApJL, 740, L13, (2011)

[9] Narayan R., McClintock J. E., Yi I., 1996, ApJ, 457, 821, (1996)

[10] Narayan R., Barret D., McClintock J. E., ApJ, 482, 448, (1997)

[11] Quataert E., Narayan R., ApJ, 520, 298, (1999)

[12] Rau A., Greiner J., Filgas R., Astron. Tel., 3140, 1, (2011)

[13] Russell, D. M., Maitra, D., Dunn, R. J. H., \& Markoff, S., MNRAS, 405, 1759, (2010)

[14] Russell, D. M., Markoff, S., Casella, P., et al. MNRAS, 429, 815, (2013)

[15] Shahbaz T., Dhillon V. S., Marsh T. R., Zurita C., Haswell C. A., Charles P. A., Hynes R. I., Casares J., MNRAS, 346, 1116, (2003)

[16] Shahbaz T., Hynes R. I., Charles P. A., Zurita C., Casares J., Haswell C. A., Araujo-Betancor S., Powell C., MNRAS, 354, 31, (2004)

[17] Shahbaz T., Dhillon V. S., Marsh T. R., Casares J., Zurita C., Charles P. A., Haswell C. A., Hynes R. I., MNRAS, 362, 975, (2005)

[18] Shahbaz T., Dhillon V. S., Marsh T. R., Casares J., Zurita C., Charles P. A., MNRAS, 403, 2167, (2010)

[19] Shahbaz T., Russell D. M., Zurita C., Casares J., Corral-Santana J. M., Dhillon V. S., Marsh T. R., 2013, MNRAS, 434, 2696, (2013)

[20] Quataert E., Narayan R., ApJ, 520, 298, (1999)

[21] Zurita C., Casares J., Shahbaz T., ApJ, 582, 369, (2003)

[22] Wright E. L., et al., AJ, 140, 1868, (2010) 